\title{
Short-term outcomes of infants with an extremely low birth weight in a resource-limited neonatal intensive care unit, Grey's Hospital, KwaZulu-Natal
}

\author{
N P Luthuli, ${ }^{1} \mathrm{MB}$ ChB, FCPaed, MMed (Paed); \\ N H McKerrow, ${ }^{1,2}$ MB ChB, BA, DCH (SA), FCPaed, MMed (Paed), PG Dip Int Res Ethics \\ ${ }^{1}$ Department of Paediatrics and Child Health, Nelson R Mandela School of Medicine, University of KwaZulu-Natal, Durban, South Africa \\ ${ }^{2}$ KwaZulu-Natal Department of Health, Durban, South Africa
}

Corresponding author: N P Luthuli (portialuthuli@ymail.com)

\begin{abstract}
Background. Neonates with an extremely low birth weight (ELBW) constitute a small proportion of live births. However, there is limited information about the outcome of this specific group in developing countries, including South Africa (SA).

Objective. To determine the outcome to discharge of ELBW neonates admitted to a resource-limited neonatal intensive care unit (NICU). Methods. A retrospective chart review was conducted of neonates admitted to the NICU at Grey's Hospital between 1 July 2011 and 30 June 2014. All neonates with a birth weight of $<1000 \mathrm{~g}$ and admitted to the unit within 24 hours of birth were included.

Results. A total of 142 neonates met the inclusion criteria. Owing to lost files or incomplete data, 105 files were analysed in the final sample. The mean birth weight was $819.1 \mathrm{~g}$ and the mean gestational age was 27.5 weeks. The survival rate to discharge was $49.5 \%$. Neonates born after 28 weeks of gestation and those with a birth weight of $>900 \mathrm{~g}$ had better outcomes but without statistical significance. There were no statistically significant associations between outcome and any maternal variables. Nasal continuous positive airway pressure ventilation was associated with higher survival, but without statistical significance.

Conclusion. The survival rate of ELBW neonates in this study is comparable to what has been reported in other developing countries, but higher than for other NICUs in SA with similar resource limitations. More studies are required to determine factors that may influence the survival rate of the ELBW neonates.
\end{abstract}

S Afr J Child Health 2019;13(3):120-124. https://doi.org/10.7196/SAJCH.2019.v13i3.1575

Millennium Developmental Goal 4 required a two-thirds reduction in under-five mortality between 1990 and $2015 .^{[1]}$ Although the number of neonatal deaths globally declined from 5.1 million to 2.5 million between 1990 and 2017, the figure still accounted for $47 \%$ of under-five deaths in 2017. ${ }^{[2]}$ The major causes of neonatal mortality are prematurity and low birth weight, asphyxia, sepsis and birth trauma. Among preterm babies, mortality and morbidity are related to gestational age and birth weight, with the highest mortality rates seen in neonates weighing $500-999$ g. ${ }^{[3}$

Neonates of an extremely low birth weight (ELBW) represent approximately $1 \%$ of live births globally. ${ }^{[4]}$ Their stay in the neonatal intensive care unit (NICU) can be protracted, which puts a burden on hospital resources. ${ }^{[5]}$ The admission of ELBW babies to the NICU remains controversial in poorly resourced settings because of the high associated morbidity, mortality and cost of care ${ }^{[6,7]}$

There is limited information about the outcome of ELBW babies in developing countries, including South Africa (SA), and what is known is restricted to larger or academic hospitals. Previous SA studies have tended to consider the outcome of babies with a very low birth weight, rather than ELBW babies. ${ }^{[8,9]}$

Kirsten et al. ${ }^{[10]}$ showed that at Tygerberg Children's Hospital, ELBW neonates with respiratory distress syndrome had a $62.9 \%$ survival rate at discharge if offered nasal continuous positive airway pressure (nCPAP) ventilation and mechanical ventilation as backup. Similarly, at the Charlotte Maxeke Johannesburg Academic Hospital (CMJAH), neonates with a birth weight of $<900 \mathrm{~g}$ not offered mechanical ventilation were reported to have a $26.5 \%$ survival rate. ${ }^{[1]}$

As there are no published reports from KwaZulu-Natal (KZN), this study investigated the outcome to discharge of ELBW neonates admitted to Grey's Hospital in Pietermaritzburg, KZN. This is a developing tertiary hospital that supports 14 district and 3 regional hospitals catering for urban, peri-urban and rural communities in the western half of the province.

\section{Methods}

A retrospective chart review was conducted on the records of all ELBW babies admitted to the NICU at Grey's Hospital, KZN between 1 July 2011 and 30 June 2014. All babies with a birth weight of $<1000 \mathrm{~g}$ and who were admitted within 24 hours of birth were included. Gestational ages were determined according to Ballard scores.

Eligible babies were identified from the NICU admission registers and the Perinatal Problem Identification Programme database. Their files were retrieved and relevant data were extracted into a spreadsheet for further analysis. Survival to discharge was the main outcome measured. SPSS version 24 (IBM Corp, USA ) was used for statistical analysis, which involved descriptive statistics for demographic data and a chi-squared test for categorical variables. A significance level of $p<0.05$ was used.

All ELBW neonates in this unit were offered basic care, which included oxygen supplementation, warmth, intravenous fluids, antibiotics, expressed breastmilk feeds and total parental nutrition if the birth weight was $>500 \mathrm{~g}$. Intermittent positive pressure ventilation (IPPV) and surfactant were offered only from 2013, at the discretion of the consultant, if birth weight was $>900 \mathrm{~g}$. Neonates with a birth weight of $>800 \mathrm{~g}$ were offered nCPAP ventilation.

\section{Results}

During the study period, 820 babies were admitted to the NICU. Of these, $142(17.3 \%)$ had a birth weight of $<1000 \mathrm{~g}$ and 141 were admitted within 24 hours of birth. As 23 files could not be found and 
incomplete data or incorrect birth weights had been recorded for another 13 babies, the final patient sample consisted of 105 files and included 9 sets of twins.

The survival rate of neonates was $49.5 \%$ (Table 1). The highest mortality rate $(73.6 \%)$ was observed in neonates born at $<28$ weeks. The highest survival rate (72.7\%) was observed in babies with a birth weight of $>900 \mathrm{~g}$

Maternal variables are shown in Table 1 . The mean age of the mothers was 26.6 years (range: 15 - 43 years). Teenage mothers accounted for $11 \%$ of the sample and $12 \%$ were older than 35 . Of the sample, $6.1 \%$ women were described as grand multiparous mothers ( $\geq 5$ deliveries) and $28.3 \%$ as primigravid mothers.

More than two-thirds of the mothers $(n=71 ; 71.7 \%)$ had visited an antenatal clinic twice or more during their pregnancy, whereas eight did not attend an antenatal clinic at all. Antenatal history was unknown for 11 mothers. Records showed that only 18 mothers had received antenatal steroids. Hypertensive disorders had been recorded for approximately half the group $(n=52 ; 52.5 \%), 16$ of whom were primigravid. Obstetric haemorrhagic disorders were recorded in six cases. Of the 99 mothers, 39 (39.4\%) were HIV infected.

There was no statistical association between the survival rate of ELBW babies and any of the maternal variables, including age, antenatal attendance, HIV status or maternal morbidities. The six babies born to grand multiparous women all survived; however, the sample size was very small $(p=0.90)$.

Neonatal outcomes in relation to neonatal variables are shown in Table 2. Only 4 of the babies in this study were outborn. Two-thirds of the babies $(n=70 ; 66.7 \%)$ were delivered via caesarean section. As determined from Ballard scores, the mean gestational age (and associated standard deviation) across the total sample was 27.5 (1.96) weeks (range 19 - 34 weeks). The mean gestational age in survivors and non-survivors was 27.5 weeks and 27.3 weeks, respectively. The overall mean birth weight was 819.1 (113.5) g (range 530 - $990 \mathrm{~g}$ ); it was 802.9 (126.1) g among survivors (range: 540 - $990 \mathrm{~g}$ ) compared with 816.7 (109.0) g (range 530 - $980 \mathrm{~g}$ ) among the non-survivors.

A 5-minute Apgar score $<7$ was recorded in 21 cases (20\%). The baby who was born before arrival did not survive. More than threequarters of the neonates $(n=83 ; 79 \%)$ were admitted within an hour of birth; 9 babies (8.6\%) were admitted after an hour or more. The time to admission was unknown in $12.4 \%$ of cases. Of those admitted more than an hour after birth, 4 (44.4\%) did not survive.

The survival rate among neonates who received nCPAP ventilation was $63.2 \%(p=0.86)$. Three of the four neonates who did not receive respiratory support survived $(p<0.00001)$. Analysis showed an overall positive association between survival rate and respiratory support in the form of nCPAP ventilation $(p=0.005)$.

Six babies $(5.7 \%)$ underwent surgical procedures. This included one laparotomy for necrotising enterocolitis; however, the baby did not survive.

The average length of stay was 25.3 days, but slightly shorter (23.7 days) in the non-survivor group. The majority of non-survivors were early neonatal deaths (Table 3), whereas 12 babies died after one week of life or more. One baby died within an hour of birth.

The overall mortality rate decreased with increasing gestational age $(p<0.0001)$ and birth weight $(p<0.001)$ (Table 2$)$.

\section{Discussion}

The survival rate of ELBW babies in this study was $49.5 \%$. There was no association between neonatal outcomes and any maternal variables. The mode of delivery, 5-minute Apgar score and time to admission were not associated with an increased survival rate of ELBW neonates.

Neonates born after 28 weeks of gestation and those with a birth weight of $>900 \mathrm{~g}$ had better outcomes, but without statistical significance. The observed trend is similar to that of comparable local studies, ${ }^{[8-12]}$ in which survival rates were also reported to improve with increasing gestational age and birth weight.

There was no association between invasive ventilatory support and outcome ( $p=0.67$ ) in our study, although a significant association was observed between non-invasive ventilatory support and outcome $(p=0.005)$.

Babies born to primigravid women had a $51.7 \%$ survival rate. A study from Iran reported a better (although not statistically significant) survival rate among babies of very low birth weight born to primigravid women. ${ }^{[13]}$

The survival rate of babies born to women with hypertensive disorders was $57.7 \%$. We hypothesised that these babies were growth restricted, which may have contributed to the better outcomes. The observed incidence of maternal hypertensive disorders (52.5\%) was similar to that reported from a study at the Groote Schuur Hospital in Cape Town, SA (54\%). ${ }^{[12]}$

In our study, survival rate did not appear to be significantly associated with antenatal care or steroids. In contrast, both Velaphi et al. ${ }^{[9]}$ and Kirsten et al. ${ }^{[10]}$ reported better neonatal outcomes when antenatal care was provided. Kalimba and Ballot ${ }^{[1]}$ similarly found that antenatal steroids improved the outcome of ELBW neonates $(p=0.06)$. The small number of women who reportedly received antenatal steroids (18\%) may be due to poor record-keeping, which could have affected the analysis.

A positive maternal HIV status did not increase the mortality rate of babies in this study. This is similar to the findings of Adhikari et al. ${ }^{[14]}$ whose study in a neonatal unit in Durban, SA, showed no difference in the survival rate of babies born to HIV-infected and HIVuninfected women; however, only $52.8 \%$ of the babies in their cohort had a birth weight of $<1500 \mathrm{~g}$. In contrast, Kalimba and Ballot ${ }^{[11]}$ reported a significantly higher risk of death in HIV-exposed ELBW babies $\left(\chi^{2}=16.34, p=0.003\right)$; however, HIV status was unknown for $45.9 \%$ of women in their study.

The neonatal survival rate (49.5\%) found in our setting is higher than that reported in similar studies at $\mathrm{CMJAH}^{[8,11]}$ and Chris Hani Baragwanath Hospital $(\mathrm{CHBH}) \cdot{ }^{[9]}$ However, the survival rate is lower than what has been reported from a comparable study at Tygerberg Hospital, where neonates were offered mechanical ventilation. It should be noted that ELBW neonates in the study at $\mathrm{CHBH}$ did not receive mechanical ventilation. The survival rate in that study improved from $32 \%$ to $41 \%$ when patients who had died in the labour ward before admission were excluded from the analysis. ${ }^{[9]}$ Ballot et al. ${ }^{[8]}$ reported a $34.9 \%$ survival rate in ELBW neonates in a study at CMJAH, in which patients with birth weights of $>900 \mathrm{~g}$ were offered nCPAP ventilation or IPPV. In a later study at the same hospital, Kalimba and Ballot ${ }^{[11]}$ reported a survival rate of $26.5 \%$ in neonates with a birth weight of $<900 \mathrm{~g}$ and who received nCPAP ventilation support in a high-care unit. In a study at Tygerberg Hospital, Kirsten et al ${ }^{[10]}$ reported a survival rate of $74.8 \%$ for ELBW neonates who were offered intubation, surfactant and immediate extubation, and then back-up mechanical ventilation. More than half of the neonates in our study received nCPAP ventilation and $63.2 \%$ of these patients survived. The survival rate of the 15 babies who received IPPV was low (33.3\%), possibly because the primary indication for IPPV is hospital-acquired infection and the majority succumbed to overwhelming sepsis.

The survival rate seen in our study is comparable to that reported from other resource-limited settings. For example, Sritipsukho et al. ${ }^{[5]}$ reported a survival rate of 52\% among ELBW neonates who were offered nCPAP ventilation in a study from Thailand. In a study from Jamaica, Trotman and Lord ${ }^{[15]}$ reported a survival rate of $43 \%$ among ELBW neonates offered mechanical ventilation. In a Chinese study, Lin et al. ${ }^{[16]}$ reported a $50 \%$ survival rate among ELBW neonates with a birth weight of $>750 \mathrm{~g}$ admitted to tertiary NICUs where 


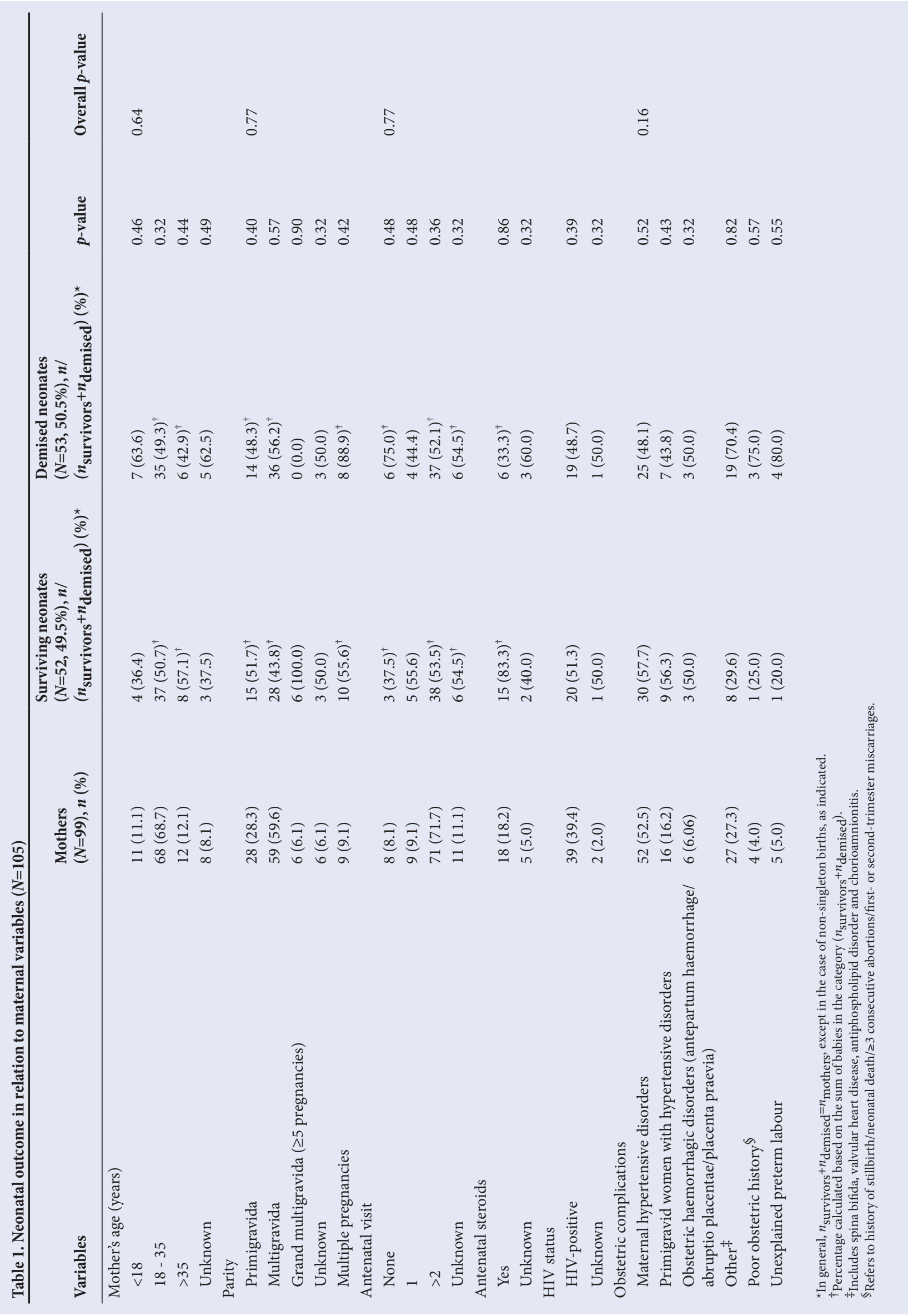




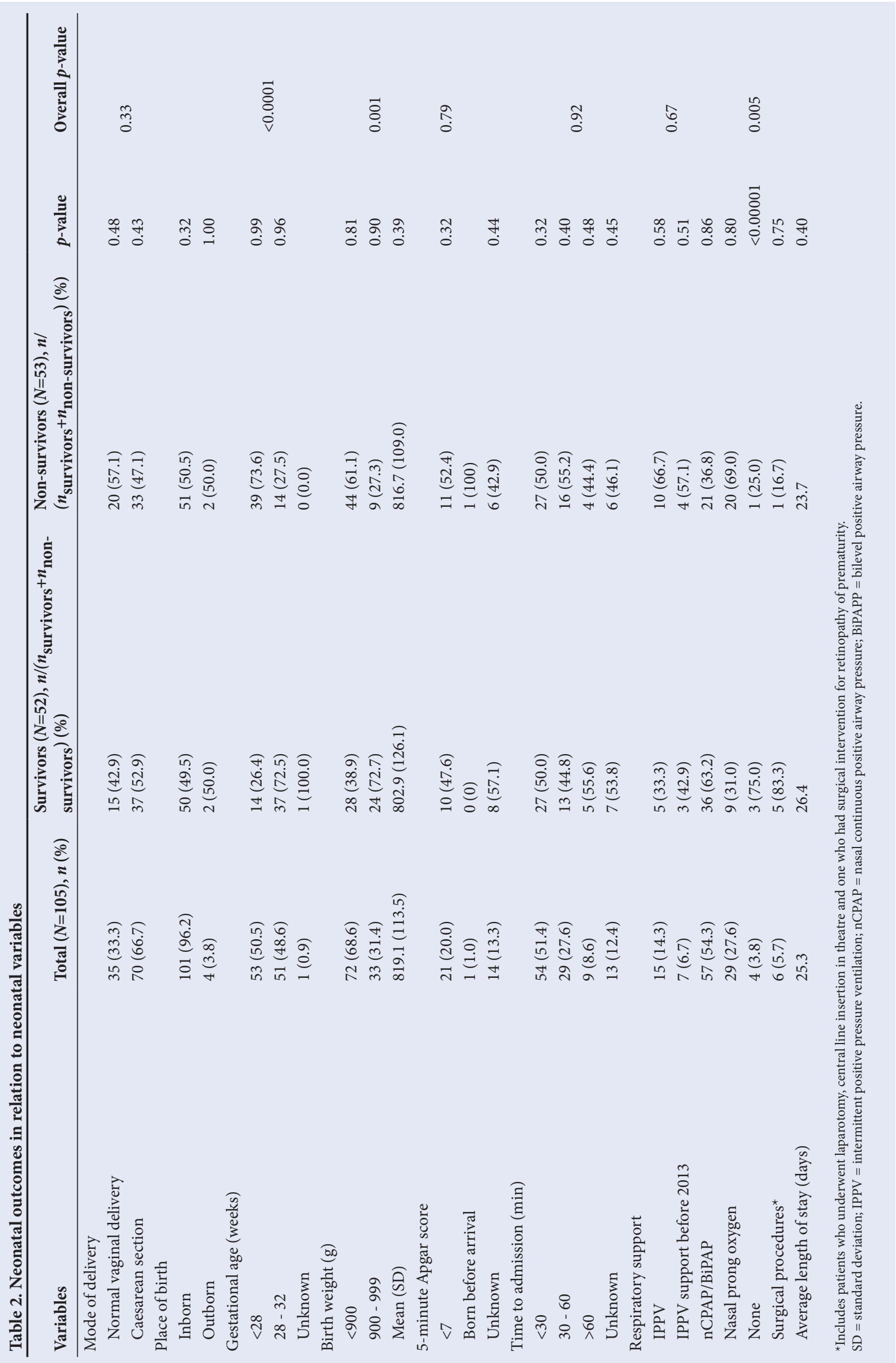




\begin{tabular}{ll}
\multicolumn{2}{l}{ Table 3. Time to death of non-survivors $(\boldsymbol{N}=\mathbf{5 3})$} \\
\hline Time & $\boldsymbol{n}(\mathbf{\%})$ \\
\hline$\leq 1$ hour & $1(1.9)$ \\
$\leq 24$ hours & $16(30.2)$ \\
$\leq 1$ week (up to day 7 of life) & $24(45.3)$ \\
$>1$ week & $12(22.6)$
\end{tabular}

nCPAP and mechanical ventilation were offered. These survival rates are, however, far below the rates $(\geq 80 \%)$ reported from betterresourced settings. ${ }^{[17]}$

\section{Study limitations}

This retrospective chart review was subject to missing data and files. As the sample was small and not considered representative, the results of this study cannot be generalised to the rest of the province or country.

\section{Conclusion}

The survival rate of ELBW neonates in our study was low, similar to the findings from comparable studies in other resource-limited settings. Further studies are needed to investigate the perinatal factors that could improve the survival rate of these neonates. In addition, further studies to determine the short- and longterm outcomes of ELBW neonates across a bigger population are recommended.

Declaration. This manuscript was submitted as a requirement for an MMed degree.

Acknowledgements. Ms Fikile Nkwanyana (Department of Biostatistics, University of KwaZulu-Natal) is thanked for assistance with statistical analysis. Author contributions. NPL was the primary investigator and responsible for data collection and analysis, and writing the manuscript. NHM supervised the study and provided critical input throughout the research process and manuscript development.

Funding. None.

Conflicts of interest. None.
1. World Health Organization (WHO). MDG 4: Reduce child mortality. https:// www.who.int/topics/millennium_development_goals/child_mortality/en/ (accessed 28 August 2017).

2. World Health Organization. Newborns: Reducing mortality. https://www.who. int/news-room/fact-sheets/detail/newborns-reducing-mortality (accessed 26 April 2018).

3. Rhoda N, Pattinson RC. Saving Babies 2012 - 2013: Ninth report on perinatal care in South Africa. Pretoria: Tshepesa Press, 2014.

4. Molteno CD, Ahrens W, Higgs SC, Malan AF, De V Heese H. Infants of very low birthweight: A follow-up study. S Afr Med J 1976;50(25):955-958.

5. Sritipsukho S, Suarod T, Sritipsukho P. Survival and outcome of very low birth weight infants born in a university hospital with level II NICU. J Med Assoc Thai 2007;90(7):1323-1329.

6. Smith J, Pieper CH, Kirsten GF. Born too soon, too small, to die - a plea for a fair innings. S Afr Med J 1999;89(11):1148-1151.

7. Prinja S, Manchada N, Mohan P, et al. Cost of neonatal intensive care delivered through district level public hospitals in India. Indian Pediatr 2013;50:839-846. https://doi.org/10.1007/s13312-013-0234-6

8. Ballot DE, Chirwa TF, Cooper PA. Determinants of survival in very low birth weight neonates in a public sector hospital in Johannesburg. BMC Paediatr 2010;10:30. https://doi.org/10.1186/1471-2431-10-30

9. Velaphi SC, Mokhachane M, Mphahlele RM, Beckh-Arnold E, Kuwanda ML Cooper PA. Survival of very-low-birth-weight infants according to birth weight and gestational age in a public hospital. S Afr Med J 2005;95(7):504-509.

10. Kirsten GF, Kirsten CL, Henning PA, et al. The outcome of ELBW infants treated with NCPAP and InSurE in a resource-limited institution. Paediatrics 2012;129(4):e952-e959. https://doi.org/10.1542/peds.2011-1365

11. Kalimba EM, Ballot DE. Survival of extremely low-birthweight infants. S Afr J Child Health 2013;7(1):13-16. https://doi.org/10.7196/sajch.488

12. Gibbs L, Tooke L, Harrison MC. Short-term outcomes of inborn v. outborn very-low-birth-weight neonates $(<1500 \mathrm{~g})$ in the neonatal nursery at Groote Schuur Hospital, Cape Town, South Africa. S Afr Med J 2017;107(10):900-903. https://doi.org/10.7196/samj.2017.v107i10.12463

13. Afjeh SA, Sabzehei MK, Fallahi M, Esmaili F. Outcome of very low birth weight infants over 3 years report from an Iranian Center. Iran J Pediatr 2013;23(5):579-587.

14. Adhikari M, Jeena P, Pillay T, Moodley A, Kieliela P, Cassol S. The HIV-1 exposed neonate: Outcome of intensive care management in the first week of life. Indian Pediatr 2005;42(12):1215-1219.

15. Trotman H, Lord C. Outcome of extremely low birthweight infants at the University Hospital of the West Indies, Jamaica. West Indian Med J 2007;56(5):409-413.

16. Lin H-J, Du L-Z, Ma X-L, et al. Mortality and morbidity of extremely low birth weight infants in the mainland of China: A multi-center study. Chin Med J (Engl) 2015;128(20):2743-2750. https://doi.org/10.4103/0366-6999.167312

17. Shim JW, Jin H-S, Bae C-W. Changes in survival rate for very-low-birthweight infants in Korea: Comparison with other countries. J Korean Med Sci 2015;30(Suppl 1):S25-S34. https://doi.org/10.3346/jkms.2015.30.s1.s25 